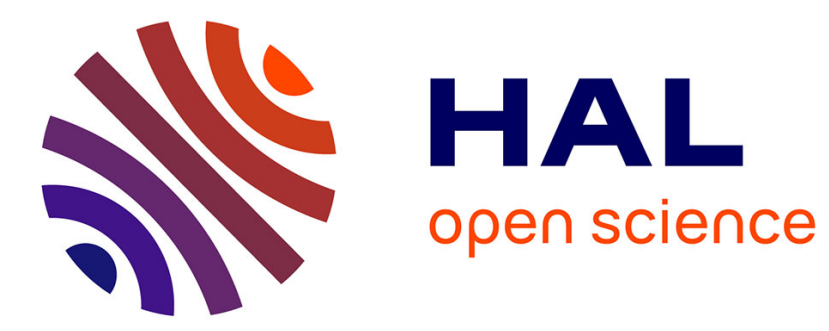

\title{
Les rapports entre les terrasses et l'évolution des versants dans les Aurès (Algérie) \\ $\mathrm{J}$ L Ballais
}

\section{To cite this version:}

J L Ballais. Les rapports entre les terrasses et l'évolution des versants dans les Aurès (Algérie). Bulletin de l'Association française pour l'étude du quaternaire, 1984, 21 (1-3), pp.170 - 172. 10.3406/quate.1984.1509. hal-01545112

\section{HAL Id: hal-01545112 \\ https://hal-amu.archives-ouvertes.fr/hal-01545112}

Submitted on 22 Jun 2017

HAL is a multi-disciplinary open access archive for the deposit and dissemination of scientific research documents, whether they are published or not. The documents may come from teaching and research institutions in France or abroad, or from public or private research centers.
L'archive ouverte pluridisciplinaire HAL, est destinée au dépôt et à la diffusion de documents scientifiques de niveau recherche, publiés ou non, émanant des établissements d'enseignement et de recherche français ou étrangers, des laboratoires publics ou privés.

\section{(ㅇ)(1) $\$$}

Distributed under a Creative Commons Attribution - NonCommercial - NoDerivatives| 4.0 


\title{
LES RAPPORTS ENTRE LES TERRASSES ET L'ÉVOLUTION DES VERSANTS DANS LES AURÈS (ALGÉRIE)
}

\author{
par J.-L. BALLAIS *
}

\begin{abstract}
RÉSUME
Dans les Aurès, les terrasses commencent à se former lors d'une phase humide. Puis, alors que leur accumulation continue, des glacis, des cônes fluvio-nivaux ou alluviaux et des coulées boueuses se mettent en place. Lors de l'aridification, se forment des croûtes calcaires, puis se déposent des sables éoliens et des croutes gypseuses.
\end{abstract}

ABSTRACT

TERRACES AND SLOPES EVOLUTION IN THE AURES MOUNTAINS (ALGERIA)

In the Aures mountains, terraces are accumulated on the course of a humid phase. During this accumulation, pediments, fans and mudslides are formed. Then, when aridity appears, calcretes, eolian sands and gypsum crusts settle.

Vers $35^{\circ}$ de latitude Nord et $6.7^{\circ}$ de longitude Est, les Aurès $(2326 \mathrm{~m})$ se placent à la charnière des deux grands ensembles qui constituent l'Atlas saharien algéro-tunisien.

\section{Caractéristiques des principales terrasses}

Dans l'ensemble, les systèmes de terrasses sont peu développés, le plus fréquemment incomplets, en particulier en altitude. Je prendrai l'exemple des terrasses du Pléistocène moyen et supérieur (terrasses III et II, la numérotation des formes se faisant à partir du lit actuel des oueds considéré comme niveau 0), les mieux représentées et qui présentent des aspects très semblables.

A l'amont des dernières cluses méridionales, les terrasses prennent un peu d'ampleur aux dépens des marnes épaisses du Campanien. Par exemple, sur l'oued Mestaoua, à Oum el Habel $(x=838,7$, $y=192,3, z=800$ ), la terrasse II, épaisse de quelques mètres, est formée de galets bien roulés, de calcaire cénomanien surtout, en lits bien nets, rele- vant vers l'aval, de petite taille $(5-10 \mathrm{~cm})$ à la base, avec des blocs $(20$ à $50 \mathrm{~cm})$ vers le haut. Ou encore, à M'Chounech, vers $320 \mathrm{~m}$ d'altitude, sur la rive gauche de l'oued el Abiod, persiste une lanière de terrasse III probable. Sa couverture, épaisse de 1 à $2 \mathrm{~m}$, comprend des blocs anguleux de calcaire maestrichtien éboulés de l'escarpement de faille surplombant, ainsi que de nombreux galets bien roulés, longs de quelques centimètres à 1 ou 2 décimètres, formés de calcaire maestrichtien et de grès et calcaires du Crétacé inférieur, dans une matrice sablo-limoneuse.

Sur les piémonts, les terrasses prennent de la puissance. Ainsi, sur l'oued el Haï, à l'aval d'El Kantara $(x=772,4, y=215,2, z=470)$, la terrasse II épaisse de 7 à $10 \mathrm{~m}$, comprend souvent :

- à la base, de gros galets atteignant jusqu'à $80 \mathrm{~cm} \times 60 \mathrm{~cm} \times 30 \mathrm{~cm}$, pris dans une matrice sableuse, calcaire (épaisseur : 1,50 m),

- au-dessus, des lentilles argilo-limoneuses, rougeâtres, ne dépassant pas $1 \mathrm{~m}$ d'épaisseur, alternant avec des lentilles grossières.

* UER des Sciences de la Terre, Université de Caen, Esplanade de la Paix, 14032 Caen Cédex (France). 
2. Les rapports avec les autres formes et formations

\subsection{Les formes et formations globalement contempo- raines}

\section{a. Les travertins :}

Nous avons montré (Ballais, Cohen, 1981) que les travertins, très souvent, sont contemporains du début de l'élaboration d'une terrasse (fig. 1) et qu'ils contiennent alors une flore indiquant un climat un peu plus humide que l'Actuel.

S

n
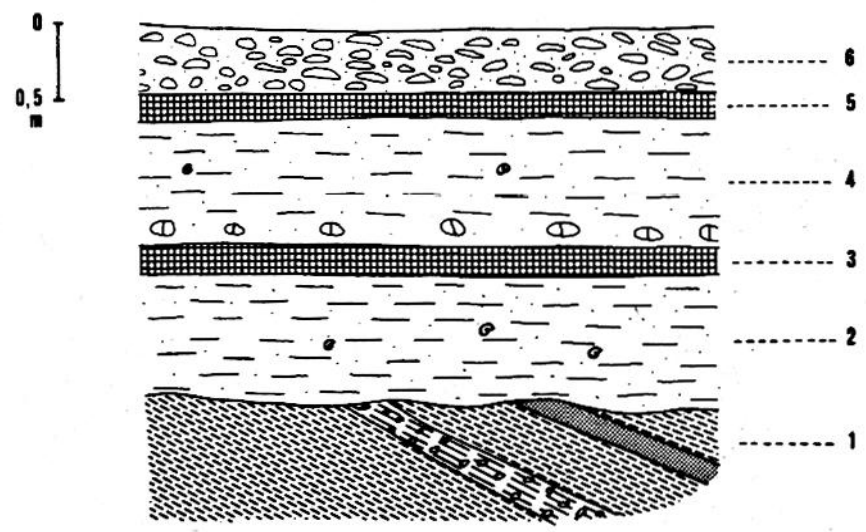

Fig. 1. - Coupe des dépôts palustres et travertins de la terrasse II d'El Barania.

1 - Substratum miocène. 2 - Argiles sableuses. 3 - Travertin. 4 - Argiles sableuses. 5 - Travertin. 6 - Galets. Echelle $=0,5 \mathrm{~m}$.

Fig. 1. - A section of terrace II at El Barania. 1 - Miocene deposits. 2 - Sandy clay. 3 - Travertine. 4 - Sandy clay. 5 - Traverine. 6 - Pebbles.

\section{b. Les coulées de solifluxion :}

Dans quelques cas, peu nombreux faute de coupe, on peut observer les modalités du passage d'une coulée de solifluxion à une terrasse. L'exemple le plus net est au $\mathbf{N}$ d'El Kantara, sur la rive gauche de l'oued el Haï, à $590 \mathrm{~m}$ d'altitude. Se succèdent, au-dessus des marnes campaniennes tronquées à l'horizontal :

- un fragment de terrasse à galets bien roulés, en lentilles interrompues, à l'amont, par une masse terreuse non litée,

- une masse amorphe de blocs de calcaire maestrichtien anguleux, parfois gigantesques (plusieurs $\mathrm{m}^{3}$ ) dans une matrice limoneuse abondante,

- un nouveau fragment de terrasse à lentilles fines alternant avec des lentilles grossières à éléments bien roulés mais comprenant aussi des blocs subanguleux, parfois énormes, très rares à la base, plus nombreux au sommet.

On peut donc en conclure que la terrasse (qui serait la III par comparaison avec la rive droite) est contemporaine d'une coulée qui se manifeste par de gros blocs anguleux mais qui, en fait, est un peu plus récente car la terrasse commence à se déposer avant que la coulée n'atteigne le talweg. Il n'est cependant pas possible d'apprécier la durée de ce décalage.

\section{c. Les glacis :}

Le raccord topographique entre glacis et terrasse est très fréquent. Cependant, il est beaucoup plus rare de pouvoir mettre en évidence le passage d'une formation à l'autre. Il faut, en effet, à la fois, que des coupes existent, mais aussi que le matériel de la terrasse soit assez différent de celui du glacis par sa morphométrie, sa pétrographie ou son altération. Ces conditions n'apparaissent guère réalisées que sur les oueds majeurs ou moyens. Le cas le plus net s'observe à l'ancien gué de l'oued el Abiod, à Arris $(x=833,2, y=221,7, z=1150)$. Là, on voit très bien s'interstratifier au milieu des galets de la terrasse II la couverture de cailloux plats et anguleux du glacis II qui constitue ensuite seule le sommet de l'accumulation. Les glacis sont donc contemporains des terrasses et un peu postérieurs.

\section{d. Les cônes fluvio-nivaux :}

Différant des simples cônes alluviaux par la part importante de la fusion de la neige dans les écoulements qui les ont construits (J.-L. Ballais, 1981, p. 201-202), les cônes fluvio-nivaux abondent dans les vallées des oueds el Haĩ, Abdi et el Abiod. Dans cette dernière, à l'aval d'Arris $(x=824,1, y=215,3$, $z=940$ ) et grâce aux coupes de la route de Biskra, on observe (fig. 2) que les gros galets roulés de la terrasse II se mélangent peu à peu, latéralement, avec des cailloux anguleux de matériel local dans une matrice fine orangée (cône II). Au sommet de la coupe, seuls les apports latéraux subsistent.

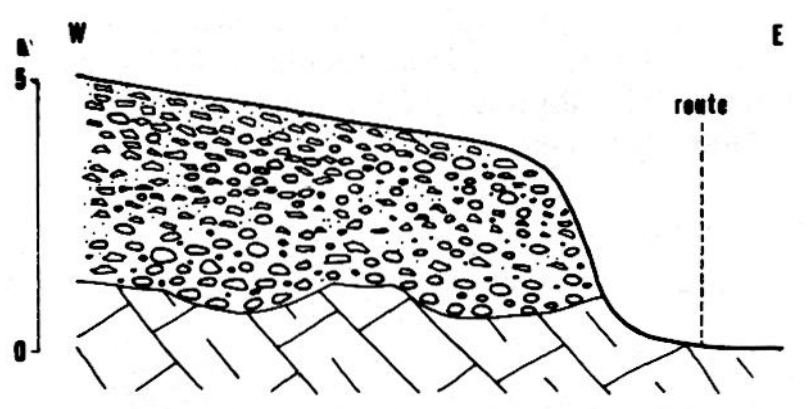

Fig. 2. - Rapports cône-terrasse daus la vallée de l'oured el Abiod.

Fig. 2. - Fan and rerrace in the valley of omed el Abiod.

Ainsi, il est établi que, à l'intérieur d'une même séquence climatique, les apports transversaux sont globalement contemporains des apports longitudinaux, mais ils se déposent un peu plus longtemps. 


\subsection{Les autres formes et formations}

a. Les dunes:

Ces rapports n'ont été observés que dans l'oued el Haĩ dont le cours aval a été le seul à être envahi par les dunes. Dans la gorge entre le djebel Hachichina et la Fontaine des Gazelles, rive droite $(x=762,2, y=206,8, z=360)$, au-dessus de la terrasse I, du sable à modelé mou (dunes à formes adoucies) a été retaillé, au sommet, par la terrasse II formée de graviers et petits galets roulés. Cette disposition se retrouve dans la terrasse I de l'oued Matraf Kebir (Ballais, Marre, Rognon, 1979).

\section{b. Les croûtes et encroûtements :}

- Les croûtes et encroûtements calcaires

Dans le quart supérieur de la terrasse III de l'oued Mestaoua, à Oum el Habel, un enduit calcaire recouvre la face inférieure des galets, alors que des feuillets de croûte calcaire s'individualisent vers le sommet du dépôt.

Au N d'El Kantara, c'est au sommet de la coulée boueuse contemporaine de la terrasse III que la matrice limoneuse abondante passe à une encroûtement calcaire blanc, pulvérulent, parfois très consolidé en surface.

Cependant, on ne peut tirer d'indications paléoclimatiques sûres de ces types de croûtes et d'encroûtements car les conditions de leur genèse ne sont pas encore bien établies, à la différence de celles des croûtes plus anciennes (Ballais, Vogt, 1981).

\section{- Les croûtes gypseuses}

Elles apparaissent dans des conditions actuellement arides, sur le piémont méridional uniquement. Parfois, comme en bordure de l'oued el Haï, au S d'El Kantara, la croûte est mélangée à un encroûtement calcaire pulvérulent, au sommet de la terrasse II. Le plus souvent, comme à M'Chounech (terrasse III) c'est une croûte gypseuse cohérente qui recouvre la terrasse.

L'étude minéralogique, granulométrique et chimique des croûtes gypseuses a montré qu'elles sont d'origine éolienne et que leur dépôt succède à celui

\section{Conclusion}

Finalement, on peut reconstituer ainsi la séquence morphoclimatique type (Ballais, Roubet, 1982) : une terrasse commence à se former, lors d'une phase humide. Puis, avec un décalage plus ou moins important, des glacis et des cônes fluvio-nivaux ou alluviaux s'élaborent, tandis que se mettent en place des coulées boueuses et que le remblaiement de la terrasse continue. Quand ce remblaiement s'interrompt, les glacis et les cônes peuvent encore fonctionner quelques temps. Ensuite se forment des croûtes calcaires puis, l'aridité croissant, se mobilisent et s'accumulent des sables éoliens. Cet épisode se termine par le dépôt de croûtes gypseuses, au maximum de l'aride, précédant immédiatement l'entaille linéaire par les oueds, lors de l'anapluvial.

\section{BIBLIOGRAPHIE}

Ballais J.-L., Marre A. et Rognon P., 1979. Périodes arides du Quaternaire récent et déplacement des sables éoliens dans les Zibans (Algérie), Rev. Géol. dyn. et Géogr. phys., Paris, vol. 21, fasc. 2, p. 97-108, 4 fig., 2 tabl., 1 pl. h.-t.

Ballais J.-L., 1981. - Recherches géomorphologiques dans les Aurès (Algérie), Thèse Doct. èsLettres, Paris I, VII-566 p., ann., index, 106 fig., XXII tabl., 107 ph. h.-t., XIII cartes coul. h.-t., multigr.

Ballais J.-L. Vogt T., 1981. - Croûtes calcaires quaternaires du piémont Nord des Aurès (Algérie), Rech. géogr. à Strasbourg, no 12, p. 23-34, 5 fig., 4 ph.

Ballais J.-L., Cohen J., 1981. - Intérêt morphogénétique et paléoclimatique des travertins des Aurès (Algérie), Coll. A.G.F. " Form. carbon. ext.", Ass. Franç. de Karst., Aix-en-Provence, mémoire no 3, p. 37-44, 4 fig., 2 tabl.

Ballais J.-L., Roubet C., 1982. - Morphogenèse et préhistoire dans les Aurès (Algérie), Rev. Géol. dyn. et Géogr. phys., Paris, vol. 23, fasc. 5, p. 375-384, 5 fig., 2 tabl. 